\title{
Isolated tuberculous epididymal mass mimicking testicular malignancy: an interesting case report and lessons learnt
}

\author{
Tejaswini Manne ${ }^{1}$, Solomon Nazareth ${ }^{1}$, Pavithra Vittalraj ${ }^{2}$, Sandhya Sundaram ${ }^{2}$, Sriram Krishnamoorthy ${ }^{1}$, Natarajan \\ Kumaresan
}

Cite this article: Manne T, Nazareth S, Vittalraj P, Sundaram S, Krishnamoorthy $\mathrm{S}$, Kumaresan $\mathrm{N}$, et al: Isolated tuberculous epididymal mass mimicking testicular malignancy: an interesting case report and lessons learnt. Ann Urol Oncol 2021; 4(1): 1-4. https://doi. org/10.32948/auo.2021.05.21

\begin{abstract}
Tuberculous epididymal mass is a condition that presents as a painless scrotal swelling. It resembles a testicular mass and is more often diagnosed after orchidectomy. About $22 \%$ of all genitourinary tuberculosis show epididymal involvement and $22 \%$ of epididymal tuberculosis are bilateral. This report reiterates the need for an increased awareness amongst the treating urologists that would enable an earlier diagnosis, appropriate treatment and may avert the need for orchidectomy in most cases.

A 35-year-old diabetic male presented with rapidly enlarging right testicle associated with recent onset of pain over the testis. He also had fever and chills. At the age of 18 , he was treated for pulmonary tuberculosis. The right testicle was enlarged, irregular and mildly tender. The right epididymis was also irregular and nodular, blended with the right testicle and indistinguishable from it. A clinical diagnosis of testicular tumour was made. Tumour markers were normal and he underwent high orchidectomy. Histopathological diagnosis confirmed right epididymal tuberculosis.

This case report mainly highlights the need for a high index of suspicion amongst the treating physicians. A previous history of treatment for pulmonary tuberculosis should alert the physician to think in lines of tuberculous pathology in epididymis too. A prompt diagnosis and early, appropriate treatment would largely prevent removal of testicles in most cases.
\end{abstract}

Key words Tuberculosis, epididymis, orchidectomy, testicular tumour, epididymal mass

\footnotetext{
1. Department of Urology, Sri Ramachandra Institute of Higher Education \& Research, Porur, Chennai 600116, India.

2. Department of Pathology, Sri Ramachandra Institute of Higher Education \& Research, Porur, Chennai 600116, India.

Correspondence: Sriram Krishnamoorthy (Department of Urology and Renal transplantation, Sri Ramachandra Institute of Higher Education \& Research Porur, Chennai 600116; Email: sriramuro@gmail.com).
} 


\section{Introduction}

Chronic, non-specific epididymal mass secondary to infective/ inflammatory pathology is not so uncommon in urological practice. Many a time, we come across patients with unilateral or bilateral testicular swelling or pain as the sole presenting symptom. Most of these patients are labelled as chronic epididymo-orchitis and are treated conservatively without further evaluation. When testicular swelling persists or if it enlarges in size, testicular malignancies are being thought of and are subjected to high inguinal orchidectomy. Tuberculous epididymitis is one condition that presents with painless enlargement of the testicle that enlarges in size and closely mimics testicular malignancy and is most often diagnosed after orchidectomy.

Genitourinary Tuberculosis (GUTB) is considered to be the most common form of extrapulmonary tuberculosis (EPTB), second only to lymph node involvement [1]. While various reports suggest GUTB accounting for up to $73 \%$ of all cases of EPTB, epididymal involvement is seen in up to $22 \%$ of all cases of GUTB [2]. Reeve reported that epididymal tuberculosis constituted up to $7 \%$ of all cases of tuberculosis [3]. Despite such a relatively common occurrence, tuberculosis of epididymis is a condition that gets often missed out in the initial evaluation process. The purpose of this case report is to emphasize the relatively widespread incidence of this entity and also to highlight the lessons we learnt after performing high inguinal orchidectomy. As this condition, if diagnosed early, can be well treated by anti-tuberculous therapy, a proper awareness amongst the treating surgeons and urologists would largely obviate the need for orchidectomy in most cases.

\section{Case report}

A 35-year-old male presented with right scrotal pain and right testicular enlargement. The enlargement was noticed over the past two weeks and the pain was more severe over the last 5 days and associated with high-grade fever, chills and rigor. $\mathrm{He}$ also had dysuria with spikes of temperature up to 102 degrees F. $\mathrm{He}$ is a known diabetic for 4 years and is on oral hypoglycemic drugs. He also received anti-tuberculous treatment for pulmonary tuberculosis, for 6 months at the age of 18 . He received 4 drugs for 2 months and 2 drugs for the next 4 months, as per the Category I regime of the Revised National Tuberculosis Control Programme (RNTCP). He was followed up for 18 months and declared to be fully cured and his subsequent sputum and chest X-ray were negative for tuberculosis.

On examination, the right testicle was enlarged and irregular. There was mild tenderness on deep palpation. A minimal reactionary secondary hydrocele was present. The right epididymis was hard and nodular and irregular in surface, indistinguishable from the right testis. However, the testicular sensation was characteristically absent. The left testicle and rest of the external genitalia were normal. Because of the rapidly enlarging size of the testicle and loss of testicular sensation, a clinical diagnosis of testicular malignancy was made.

The absolute leucocyte count was 14,400 cells/cub mm. His Serum Creatinine was $1.3 \mathrm{mg} \%$. Urine microscopy and urine culture were normal. Tumour markers alpha-fetoprotein, beta HCG and Lactic dehydrogenase levels were normal. Colour Doppler Ultrasonogram (USG) scrotum revealed a $6 \mathrm{~cm}$ sized large irregular heteroechoic mass in the right testicle, with multiple areas of hypoechogenicity within it, consistent with bleeding or necrosis within the testicular mass (Fig 1a). The mass showed increased vascularity within it. Tunica albuginea was ill-defined. The epididymis was also enlarged and densely adherent to the testicle and indistinguishable from the right testicle. Minimal reactionary hydrocele was seen.
Given a demonstrable testicular lesion both clinically and sonologically with normal tumour marker levels, a final diagnosis of a seminomatous testicular tumour was made. The pain in the testicle was suspected to be due to internal bleeding within the mass. His HIV test results were negative. After optimizing, the patient underwent the right high Inguinal Orchidectomy. Intraoperatively, the testicle was densely adherent to the epididymis, with no plane of cleavage between the two. However, the cut surface did not show any evidence of solid mass but had only small pockets of abscess cavities.

Fig 1b illustrates the high orchidectomy specimen, where the testis, epididymis, cord structures and the fascia around the testicles were removed en mass. Cut section of the excised specimen revealed an enlarged testicle, weighing 65 grams. The testicle was measuring $5 \times 4.5 \times 3 \mathrm{cms}$, with areas of abscess cavities within the parenchyma. The tunica vaginalis was densely adherent and inseparable from the epididymis. The whole epididymis was enlarged and irregular with a dull grey lesion and areas of caseous necrosis within the epididymal tissue. The lesion was found to extend from the epididymis onto the sinus of the testicle (Fig 1c, blue arrow).

Fig 1d illustrates the microscopic examination of the testicle with a dense inflammatory infiltrate composed of neutrophils with few areas of necrosis. There was no evidence of a testicular tumour or tuberculosis. Epididymis showed inflammatory cell infiltration with epithelioid granulomas and Langhans giant cells, consistent with tuberculosis (Fig 1e). However, special stains for Acid Fast Bacilli (AFB) were negative (Fig 1f).

After orchidectomy, the patient was started on Category I regime anti-tuberculous treatment. He was given 4 drugs for 2 months and is currently taking 2 drugs for 4 months, as per RNTCP protocol. After completion of 6 months of therapy, he would be advised to be on follow-up for 18 months, to ascertain that he has achieved a disease-free state. He would also be forewarned to be on lifelong surveillance for a possible reactivation of the bacilli, later on in life.

\section{Discussion}

Chronic epididymo-orchitis is one of the common inflammatory conditions affecting the testicles. Epididymal TB, which closely mimics inflammatory pathology of the epididymis, is believed to be due to a retrograde spread from tuberculosis of the prostate or secondary to renal tuberculosis $[4,5]$. Isolated TB Epididymitis (ITE) is defined as tuberculous epididymitis without clinical and laboratory evidence of renal involvement. It is rare and difficult to diagnose. ITE can also be either associated with the onset of HIV infection or caused by intravesical BCG instillation in cases of bladder carcinoma [6].

ITE usually presents as a painful scrotal swelling but can also present as a painless scrotal mass mimicking malignancy. Storage urinary symptoms that are commonly seen in acute epididymoorchitis are not associated with ITE. ITE is often unilateral, but bilateral cases are reported [7, 8]. In his study on 18 patients with tuberculous epididymitis, Chung et al report an overall bilateral incidence in $22 \%$ of individuals [9]. Epididymal tuberculosis first appears in the tail of the epididymis due to its rich blood supply and gradually ascends to the head and finally affecting the entire epididymis.

ITE is reported to affect children too. Such lesions have a higher degree of involvement of the tail of epididymis in children due to hematogenous spread, while adults have a higher chance of development of epididymo-orchitis due to ascending infection from the urinary tracts [10].

Granulomatous inflammation is typically initiated in most cases that gradually destroys and replaces the entire epididymis with 

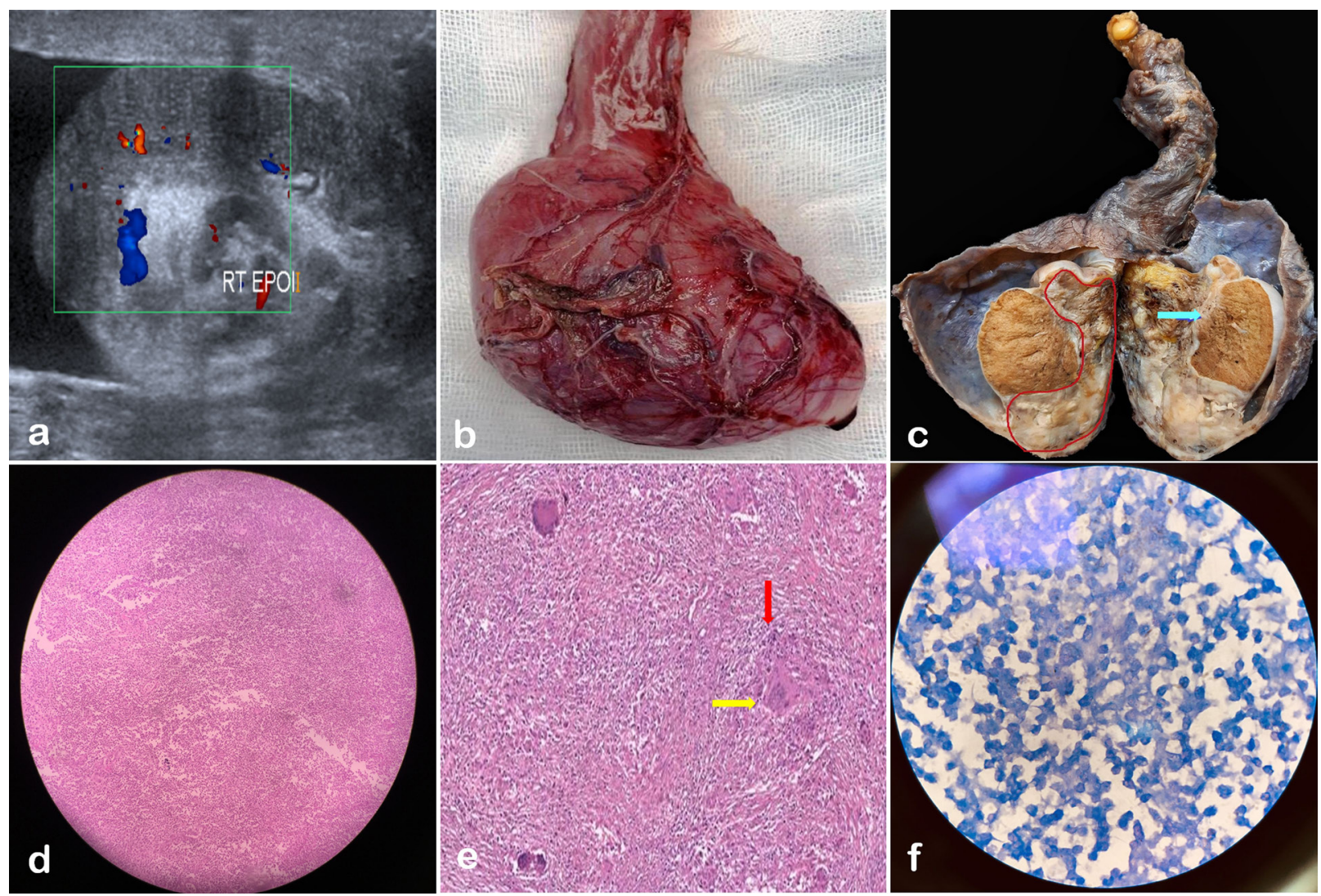

Figure 1. Illustration of isolated tuberculous epididymitis. a: Colour Doppler Ultrasonogram of scrotum showing ill-defined hetero-echoic epididymal mass compressing the testicular parenchyma. b: High orchidectomy specimen with the testicle, epididymis and spermatic cord removed. c: Cut section of orchidectomy specimen showing the irregularly enlarged epididymis (red line) and extension of the lesion into the sinus of testicle (blue arrow). d: Microphotograph of the testicle showing Hematoxylin-Eosin staining, 40X magnification, with sheets of necrosis and dense inflammatory infiltrate composed of abundant neutrophils. Normal testicular architecture is not seen. e: Microphotograph of the epididymis, 100X magnification, showing epitheloid granulomas (red arrow) and Langhan's giant cells (yellow arrow). f: Ziehl-Nielson staining, 400X magnification, showing absence of acid-fast bacilli.

granulomas, Langhans giant cells and chronic inflammatory cell infiltrates [11]. In our case, a fine needle aspiration biopsy (FNAB) might have clinched the diagnosis, but fear of needle track seedling or scrotal lymphatic violation prevented us from doing a needle biopsy. Moreover, as this patient had a rapidly enlarging testicular mass with absent sensation, the diagnosis was more in favour of a malignant tumour of the testicle. Sah et al, in their study on 40 cases of tuberculous epididymitis concluded that FNAB plays a prime role in making a diagnosis of epididymal tuberculosis and obviates the need for orchidectomy in most cases [12]. But FNAB alone may not clinch the diagnosis in all patients. Parekh DJ, in his expert comments to Viswaroop et al's study, observed that a significant number of cases are likely to be missed out by FNAB alone, and recommended a formal biopsy of the epididymis in such cases. He also observed that epididymal neoplasms are to be borne in mind when we deal with such cases [13].

Doppler USG shows an enlarged epididymis with variable echotexture. It may be either hypoechoic or hyperechoic. Granulomatous masses can be very firm and blend with the adjacent testicles, making them indistinguishable and inseparable. Occasionally, differentiating the epididymal mass from a primary testicular mass may be difficult. Our patient also had a similar epididymal mass that could not be distinguished in clinical or sonological evaluation. Though our patient, with a strong history of treatment for tuberculosis, had many strong clinical signs and symptoms pointing towards a diagnosis of tubercular aetiology, the loss of testicular sensation and heteroechogenecity in ultrasound scrotum made us think of testicular tumour ahead of other differential diagnoses.

Doppler ultrasound is the first choice of investigation for epididymal tuberculosis. CT and MRI have very limited added value. It is often possible to make a clear diagnosis by examining for acid-fast bacilli in samples of ruptured tissue through pus or secretion smears. In solid masses, diagnosis often gets delayed. Epididymal tuberculosis should be highly suspected when patients have persistent or recurrent epididymitis, not responding to antibiotics. A hypovascular central area with a hypervascular peripheral lesion correlates well with granuloma with central caseation necrosis and peripheral vascularity [14, 15]. In one of the largest series on 188 patients who underwent scrotal ultrasonogram, Riftkin et al studied 36 patients who had inflammatory or neoplastic lesions of the scrotum. In their study, they concluded that differentiating neoplastic and inflammatory masses of testicles may not be always possible with ultrasonogram and surgical exploration may be often needed in many cases [16 Riftkin]. Marco et al, in their review of seminoma of testicles and its mimickers, observed that the radiologists play a critical role in making a diagnosis of seminoma and a sound basic understanding of the clinical, pathological and radiological details will greatly aid them to contribute to high-quality care to the affected patients [17]. 
ITE responds well to anti-tubercular therapy. Anti-tuberculosis treatment with rifampicin, isoniazid, pyrazinamide, or ethambutol for 3-6 months is usually recommended [18]. Surgical intervention is recommended in non-responders to 2 months of anti-tuberculous treatment [19]. Epididymo orchidectomy is usually reserved for patients who do not respond to medical therapy.

\section{Conclusions}

A retrospective analysis of our case study shows that a prior history of treatment for tuberculosis in childhood, doppler findings of central hypoechogenicity and a common endemicity of tuberculosis in this part of the world were all indicators of tuberculous aetiology. However, the rapid growth of the mass and a loss of testicular sensation were misleading and made us perform orchidectomy. An FNAB could have obviated the need for orchidectomy. A high index of clinical suspicion is mandatory in making a diagnosis of tubercular epididymitis. A simple urine smear for AFB or AFB culture of urine could have clinched the diagnosis of TB epididymis. Axial imaging using CT or MRI scan would have also given more clarity on the diagnosis.

\section{Acknowledgements}

NIL

\section{Ethical policy}

Approval was taken from institutional ethical committee. The study was performed in accordance with the Declaration of Helsinki. Patients gave their informed consent for their participation.

\section{Author contributions}

All authors have equally contributed to the manuscript. TM \& SN assisted SK in performing the surgery. PV and SS helped in giving the specimen, a pathological diagnosis. NK helped in preparing the manuscript.

\section{Competing interests}

NIL

\section{Funding}

NIL

\section{References}

1. Kapoor R, Ansari MS, Mandhani A, Gulia A: Clinical presentation and diagnostic approach in cases of genitourinary tuberculosis. Indian J Urol 2008, 24(3): 401-405.

2. Viswaroop B S, Kekre N, Gopalakrishnan G: Isolated tuberculous epididymitis: A review of forty cases. J Postgrad Med 2005, 51: 109111 .

3. Reeve HR, Weinerth JL, Peterson LJ: Tuberculosis of epididymis and testicle presenting as hydrocele. Urology 1974, 4: 329-31.

4. Das A, Batabyal S, Bhattacharjee S, Sengupta A: A rare case of isolated testicular tuberculosis and review of literature. J Family Med Prim Care 2016, 5(2): 468-470.

5. Wise GJ, Shteynshlyuger A: An update on lower urinary tract tuberculosis. Curr Urol Rep 2008, 9(4): 305-313.

6. Pérez-Jacoiste Asín MA, Fernández-Ruiz M, López-Medrano F, Lumbreras C, Tejido Á, San Juan R, Arrebola-Pajares A, Lizasoain M, Prieto S, Aguado JM: Bacillus Calmette-Guérin (BCG) infection following intravesical BCG administration as adjunctive therapy for bladder cancer: incidence, risk factors, and outcome in a singleinstitution series and review of the literature. Medicine (Baltimore). 2014, 93(17): 236-254.

7. Wankhar B, Batchala PP, Sailo S: Case report: USG of bilateral tuberculous epididymo-orchitis. Indian J Radiol Imaging 2008, 18(1): 76-79.

8. Krieger JN: Epididymitis, orchitis, and related conditions. Sex Transm Dis 1984, 11(3): 173-181.

9. Chung JJ, Kim MJ, Lee T, Yoo HS, Lee JT: Sonographic findings in tuberculous epididymitis and epididymo-orchitis. J Clin Ultrasound 1997, 25(7): 390-394.

10. Cabral DA, Johnson HW, Coleman GU, Nigro M, Speert DP: Tuberculous epididymitis as a cause of testicular pseudomalignancy in two young children. Pediatr Infect Dis 1985, 4(1): 59-62.

11. Badmos KB: Tuberculous epididymo-orchitis mimicking a testicular tumour: a case report. Afr Health Sci 2012, 12(3): 395-397.

12. Sah SP, Bhadani PP, Regmi R, Tewari A, Raj GA: Fine needle aspiration cytology of tubercular epididymitis and epididymoorchitis. Acta Cytol 2006, 50(3): 243-249.

13. Parekh D J: Isolated tuberculous epididymitis. J Postgrad Med 2005, 51: 111.

14. Park KW, Park BK, Kim CK, Lee HM, Oh YL: Chronic tuberculous epididymo-orchitis manifesting as a non-tender scrotal swelling: magnetic resonance imaging-histological correlation. Urology 2008, 71(4): 755.e5-7.

15. Yang DM, Yoon MH, Kim HS, Jin W, Hwang HY, Kim HS, Cho SP, Kim DS: Comparison of tuberculous and pyogenic epididymal abscesses: clinical, gray-scale sonographic, and color Doppler sonographic features. AJR Am J Roentgenol 2001, 177(5): 1131-1135.

16. Rifkin MD, Kurtz AB, Pasto ME, Rubenstein JB, Cole-Beuglet C, Baltarowich O, Goldberg BB: The sonographic diagnosis of focal and diffuse infiltrating intrascrotal lesions. Urol Radiol 1984, 6(1): 20-26.

17. Marko J, Wolfman DJ, Aubin AL, Sesterhenn IA: Testicular Seminoma and Its Mimics: From the Radiologic Pathology Archives. Radiographics 2017, 37(4): 1085-1098.

18. Man J, Cao L, Dong Z, Tian J, Wang Z, Yang L: Diagnosis and treatment of epididymal tuberculosis: a review of 47 cases. PeerJ 2020, 8: e8291.

19. Gow JG, Barbosa S: Genitourinary tuberculosis. A study of 1117 cases over a period of 34 years. Br J Urol 1984, 56(5): 449-455. 